OPEN ACCESS

Edited by:

Hendrik Tevaearai Stahel, Bern University Hospital, Switzerland

Reviewed by: Mohamad Bashir, SRM University, India Mohammed Idhrees,

SIMS Hospital, India

*Correspondence:

Xiaofan Huang dr_xfhuang@hust.edu.cn Xinling Du

xinlingdu@hust.edu.cn

Anchen Zhang

644720889@qq.com Ping Ye

blue314@163.com

†These authors have contributed equally to this work

Specialty section: This article was submitted to Heart Surgery,

a section of the journal

Frontiers in Cardiovascular Medicine

Received: 22 September 2021 Accepted: 30 November 2021 Published: 23 December 2021

Citation:

Wang D, Le S, Luo J, Chen X, Li R, Wu J, Song $Y$, Xie F, Li X, Wang $H$, Huang $X$, Ye P, Du X and Zhang A (2021) Incidence, Risk Factors and

Outcomes of Postoperative Headache After Stanford Type a Acute Aortic Dissection Surgery. Front. Cardiovasc. Med. 8:781137. doi: 10.3389/fcvm.2021.781137

\section{Incidence, Risk Factors and Outcomes of Postoperative Headache After Stanford Type a Acute Aortic Dissection Surgery}

\author{
Dashuai Wang ${ }^{1 \dagger}$, Sheng Le ${ }^{1 \dagger}$, Jingjing Luo ${ }^{1 \dagger}$, Xing Chen ${ }^{1 \dagger}$, Rui $\mathrm{Li}^{1 \dagger}$, Jia $\mathrm{Wu}^{2}$, Yu Song ${ }^{1}$, \\ Fei Xie ${ }^{3}$, Ximei $\mathrm{Li}^{4}$, Hongfei Wang ${ }^{1}$, Xiaofan Huang ${ }^{1 *}$, Ping $\mathrm{Ye}^{5 *}$, Xinling $\mathrm{Du}^{1 *}$ and \\ Anchen Zhang ${ }^{5 *}$
}

\begin{abstract}
${ }^{1}$ Department of Cardiovascular Surgery, Union Hospital, Tongji Medical College, Huazhong University of Science and Technology, Wuhan, China, ${ }^{2}$ Key Laboratory for Molecular Diagnosis of Hubei Province, The Central Hospital of Wuhan, Tongji Medical College, Huazhong University of Science and Technology, Wuhan, China, ${ }^{3}$ Department of Cardiovascular Surgery, First Affiliated Hospital of Zhengzhou University, Zhengzhou, China, ${ }^{4}$ Department of Nursing, Huaihe Hospital of Henan University, Kaifeng, China, ${ }^{5}$ Department of Cardiology, The Central Hospital of Wuhan, Tongji Medical College, Huazhong University of Science and Technology, Wuhan, China
\end{abstract}

Background: Postoperative headache $(\mathrm{POH})$ is common in clinical practice, however, no studies about $\mathrm{POH}$ after Stanford type A acute aortic dissection surgery (AADS) exist. This study aims to describe the incidence, risk factors and outcomes of POH after AADS, and to construct two prediction models.

Methods: Adults who underwent AADS from 2016 to 2020 in four tertiary hospitals were enrolled. Training and validation sets were randomly assigned according to a 7:3 ratio. Risk factors were identified by univariate and multivariate logistic regression analysis. Nomograms were constructed and validated on the basis of independent predictors.

Results: $\mathrm{POH}$ developed in 380 of the 1,476 included patients (25.7\%). Poorer outcomes were observed in patients with $\mathrm{POH}$. Eight independent predictors for $\mathrm{POH}$ after AADS were identified when both preoperative and intraoperative variables were analyzed, including younger age, female sex, smoking history, chronic headache history, cerebrovascular disease, use of deep hypothermic circulatory arrest, more blood transfusion, and longer cardiopulmonary bypass time. White blood cell and platelet count were also identified as significant predictors when intraoperative variables were excluded from the multivariate analysis. A full nomogram and a preoperative nomogram were constructed based on these independent predictors, both demonstrating good discrimination, calibration, clinical usefulness, and were well validated. Risk stratification was performed and three risk intervals were defined based on the full nomogram and clinical practice.

Conclusions: $\mathrm{POH}$ was common after AADS, portending poorer outcomes. Two nomograms predicting $\mathrm{POH}$ were developed and validated, which may have clinical utility in risk evaluation, early prevention, and doctor-patient communication.

Keywords: headache, cardiac surgery, Stanford type A acute aortic dissection, risk factor, nomogram 


\section{INTRODUCTION}

Stanford type A acute aortic dissection is a life-threatening cardiovascular disease related to significant risk of morbidity and mortality (1). Although great improvement has been made in diagnostic techniques and initial management over the past decades, prompt surgical interventions remain the standard treatment (2). However, survival after Stanford type A acute aortic dissection surgery (AADS) is still suboptimal and a considerable proportion of patients develop various postoperative complications (1).

Postoperative headache (POH) is one of the most common surgical complications, which is associated with decreased quality of life, poorer outcomes, and additional economic burden (3-7). At present, many studies describing the incidence and outcomes of $\mathrm{POH}$ have been conducted and several independent risk factors for $\mathrm{POH}$ have been reported in the literature (7-12). Nevertheless, none of these studies were carried out in patients undergoing AADS and available information is still lacking in this population.

In the present study, we aimed to investigate the incidence, risk factors and outcomes of $\mathrm{POH}$ in adult patients who underwent AADS, and to construct and validate two nomogram models for POH after AADS to provide help for risk assessment and early prevention.

\section{MATERIALS AND METHODS}

\section{Ethical Statement}

This study was conducted according to the ethical statement of the Declaration of Helsinki. The Ethics Committee of Tongji Medical College of Huazhong University of Science and Technology (IORG No. IORG0003571) approved this study. Written informed consent was waived due to its observational, retrospective nature.

\section{Study Population}

This was a multicenter, observational, retrospective study. Consecutive adult patients (older than 18 years) who underwent AADS in four tertiary care centers between 2016 and 2020 were enrolled. Patients with the following conditions were excluded from the study: (1) intraoperative death or postoperative unconsciousness, and (2) records with missing data.

\section{Data Collection and Variables}

We collected clinical data using the hospital's electronic medical record management systems. Pre-, intra-, and post-operative variables were collected and analyzed. Preoperative variables included sex, age, body mass index, smoking, drinking, diabetes mellitus, hypertension, cerebrovascular disease, chronic headache history, chronic obstructive pulmonary disease, gastrointestinal tract disease, peripheral vascular disease, atrial fibrillation, left ventricular ejection fraction, cardiac function,

\footnotetext{
Abbreviations: AADS, Stanford type A acute aortic dissection surgery; AUC, area under the receiver operating characteristic curve; $\mathrm{CI}$, confidence interval; $\mathrm{CPB}$, cardiopulmonary bypass; DHCA, deep hypothermic circulatory arrest; ICU, intensive care unit; $\mathrm{OR}$, odds ratio; $\mathrm{POH}$, postoperative headache; $\mathrm{RBC}$, red blood cell; ROC, receiver operating characteristic; WBC, white blood cell.
}

pulmonary artery hypertension, pericardial effusion, general surgery history, cardiac surgery history, red blood cell (RBC) count, white blood cell (WBC) count, platelet count, hemoglobin, serum creatinine, urea nitrogen, uric acid, albumin, and globulin levels. Intraoperative variables included cardiopulmonary bypass (CPB) time, aortic cross clamp time, use of deep hypothermic circulatory arrest (DHCA), and transfusion of RBC. Postoperative variables included readmission to intensive care unit (ICU), reintubation, tracheotomy, mortality, ICU duration, and hospital stay.

\section{Definitions of Important Variables}

In this study, $\mathrm{POH}$ was diagnosed on the basis of a self-reported or recorded headache identified in the electronic medical records. Body mass index was calculated on the basis of height and body weight. Smoking history referred to current or previous daily smoking. Chronic obstructive pulmonary disease was defined in accordance with previous diagnosis, self-report, or FEV1/FVC $\leq 0.7$. Chronic headache history referred to recorded or self-reported migraine or other kinds of recurrent headaches. Cerebrovascular disease referred to a history of carotid artery surgery, transient ischemic attack, cerebral infarction, cerebral hemorrhage or stroke. Diabetes mellitus referred to fasting glucose $\geq 7.0 \mathrm{mmol} / \mathrm{L}$, random glucose $\geq 11.1 \mathrm{mmol} / \mathrm{L}$, or previous diagnosis of diabetes mellitus. Hypertension referred to previous diagnosis of hypertension or blood pressure > 140/90 $\mathrm{mmHg}$.

\section{Statistical Analysis}

Patients were divided into the training set and the validation set by 7:3 ratio. The development and internal validation of the model was performed using the training set and the external validation of the model was performed using the independent validation set. Normally distributed continuous variables were presented as means with standard deviations. Non-normally distributed continuous variables were presented as medians with inter-quartile ranges. Categorical variables were presented as frequencies with percentages. We first performed univariate logistic regression analysis to screen possible risk factors. Factors with $P<0.1$ or considered to be clinically significant were further entered into a forward stepwise multivariate logistic regression analysis to identify independent risk factors. A nomogram based on these independent risk factors was then constructed.

We performed internal validation by bootstrap method using 1,000 replicates in the training set and external validation in the validation set. The area under the receiver operating characteristic (ROC) curve (AUC) was used to assess the discrimination. Both Hosmer-Lemeshow goodness-of-fit test and visual inspection were used to assess the calibration. Decision curves and clinical impact curves were used to assess the clinical usefulness. The Delong method was used to compare the AUCs between the training and the validation sets (13).

Statistical analyses were performed using SPSS (IBM SPSS Statistics 26.0, SPSS Inc., Chicago, IL) and R software (version 4.0.5, www.R-project.org/). A two-tailed $P$-value $<0.05$ was deemed statistically significant. 


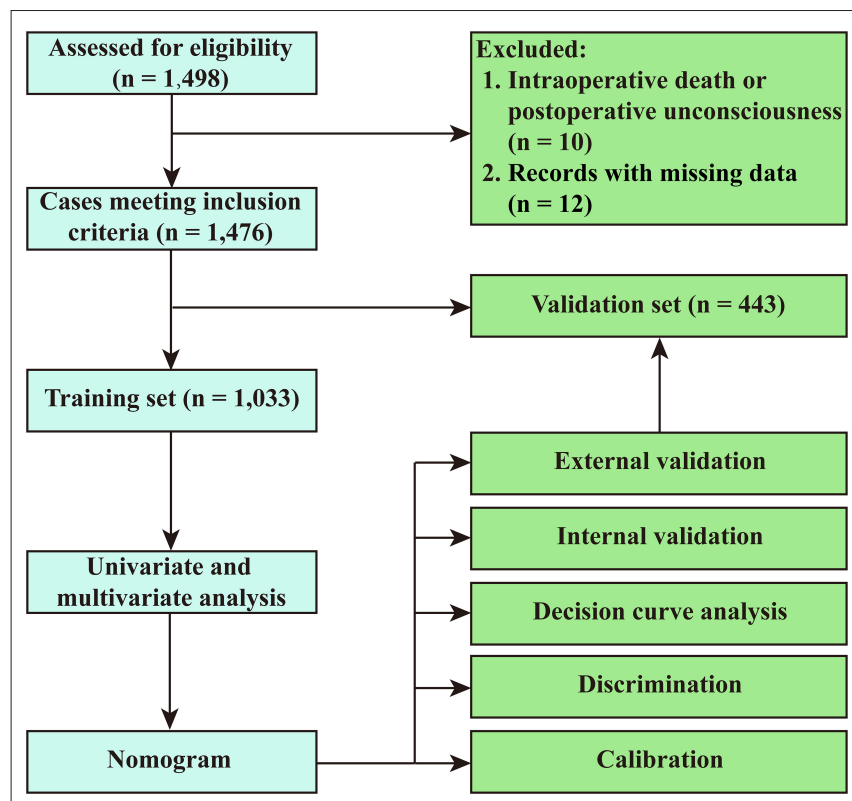

FIGURE 1 | Flow chart of the study.

\section{RESULTS}

\section{Demographic Characteristics}

Among the 1,498 adults who underwent AADS, 10 patients died intraoperatively or lapsed into unconsciousness postoperatively, and 12 patients had missing data in the medical records (Figure 1). The remaining 1,476 patients meeting the inclusion criteria were divided into two groups based on if one or two episodes of headache developed during their postoperative hospitalization and were further analyzed. The mean age of these included patients was $50.83 \pm 11.35$ years, $75.6 \%$ were men. The overall morbidity of POH after AADS was $25.7 \%$.

There were multiple comorbidities and underlying conditions in this study population, in which smoking history existed in $43.9 \%$ of the patients, drinking history in $35.8 \%$, hypertension in $68.1 \%$, diabetes mellitus in $4.3 \%$, cerebrovascular disease in $17.8 \%$, chronic headache history in $11.4 \%$, peripheral vascular disease in $13.6 \%$, gastrointestinal tract disease in $8.5 \%$, chronic obstructive pulmonary disease in $1.0 \%$, atrial fibrillation in $0.8 \%$, general surgery history in $20.5 \%$, cardiac surgery history in $6.5 \%$, pulmonary artery hypertension in $2.8 \%$, pericardial effusion in 27.0\%. The median CPB time was $204(166,247)$ min, aortic cross clamp time was $116(94,143)$ min, intraoperative RBC transfusion was $4.0(2.5,6.0)$ units, and DHCA was used in $58.9 \%$ of the patients. No significant difference was observed with regard to baseline conditions and operative variables between the training set and the validation set (Table 1).

\section{Development of the Full Nomogram}

The results of the univariate analysis conducted in the training set are presented in Table 2. Factors with $P<$ 0.1 or considered to be clinically significant were further entered into a forward stepwise multivariate logistic regression analysis to identify independent risk factors. Co-linearity of covariates were assessed and highly collinear covariates were removed from the model. Finally, eight independent risk factors were identified in the full model, including younger age, female sex, smoking history, cerebrovascular disease, chronic headache history, the use of DHCA, CPB time, and intraoperative $\mathrm{RBC}$ transfusion (Table 3). A full nomogram used to predict the probability of $\mathrm{POH}$ after AADS was then constructed based on these preoperative and intraoperative predictors (Figure 2). The nomogram scaled each regression coefficient to a scale of $0-100$ points, which demonstrated their relative importance. We also created an interactive web-based dynamic nomogram which is available online (https://xinlingdu. shinyapps.io/dynnomapp/).

The probability range of $\mathrm{POH}$ after AADS predicted by the nomogram was large. The personalized risk can be directly and easily assessed by summing the points of all the predictors. Young females who had a history of smoking, cerebrovascular disease, chronic headache, longer $\mathrm{CPB}$ time, more intraoperative $\mathrm{RBC}$ transfusion, and experienced DHCA may obtain more points and thus at a higher risk of $\mathrm{POH}$. A concrete case is illustrated in Figure 2. For the online predictive system, press the "Quit" button in the bottom-left corner to exit the application and reload the procedure. Fill in the information of a concrete patients and click the "Predict" button, the predicted probability of $\mathrm{POH}$ after AADS was presented in the "Graphical summary" area on the right. The information of the patient and the model can also be acquired by clicking the "Numerical summary" and "Model summary” (Supplementary Figure 1).

\section{Validation and Assessment of the Full Nomogram}

The nomogram was well validated by both internal and external validations. By visual inspection, the calibration curves showed good consistency between estimated and actual probabilities. This was in agreement with the results of the goodness-offit test, with Hosmer-Lemeshow chi-square statistics of 5.226 $(P=0.733$, Figure 3A) and $3.134(P=0.926$, Figure 3B $)$ in the training and validation sets. ROC curves were plotted to assess the discrimination, and the AUCs were 0.842 [95\% confidence interval (CI), 0.815-0.869] and 0.847 (95\% CI, 0.806-0.888) in the training and validation sets (Figure 3C), both indicating excellent predictive capability. There was no significant difference between the two AUCs $(P=0.83)$. Decision curve analysis was carried out to evaluate the clinical usefulness of the nomogram. The decision curves indicated that the nomogram could obtain more net benefits across a wide range of threshold probabilities than either the treat-none scheme or the treat-all-patients scheme both in the training and validation sets (Figure 3D). The clinical impact curves also revealed that the nomogram was clinically useful (Figures 3E,F).

\section{Development, Validation, and Assessment of the Preoperative Nomogram}

The above nomogram model was established using both preoperative and intraoperative variables. To facilitate early 
TABLE 1 | Comparison of characteristics between the training and validation sets.

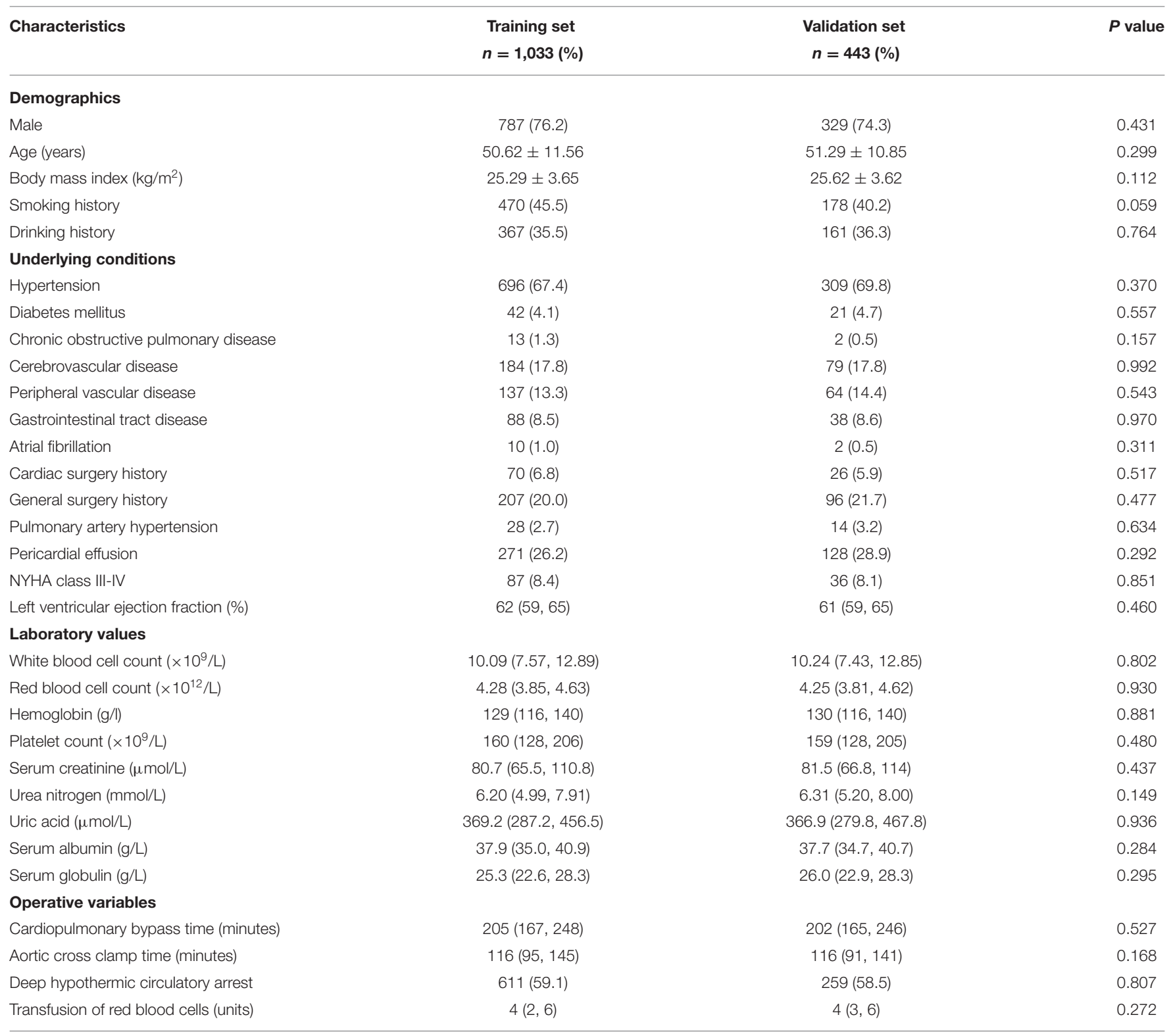

preoperative prediction, we further established a preoperative nomogram using only preoperative factors. Seven independent predictors were identified by multivariate logistic regression analysis in the training set, including lower platelet count, higher WBC count, and the five preoperative predictors mentioned above (Table 4). Then, a preoperative nomogram was constructed on the basis of these predictors (Figure 4A).

This nomogram was also well validated by both internal validation using bootstrap method in the training set and external validation in the independent validation set. The model fitted well by visual inspection of the calibration curves and goodnessof-fit test, with Hosmer-Lemeshow chi-square statistics of 7.998 $(P=0.434$, Figure 4B) and $10.148(P=0.255$, Figure 4C) in the training and validation sets, respectively. The AUCs were respectively 0.740 (95\% CI, $0.704-0.775)$ and 0.760 (95\% CI, $0.705-0.814)$ in the training and validation sets, indicating no significant difference $(P=0.55$, Figure 4D). The decision and clinical impact curves also demonstrated that the nomogram may have usefulness in clinical practice.

\section{Risk Stratification}

We further performed a risk stratification on the basis of the full nomogram and clinical practice (Table 5). We selected predicted probabilities of 0.1 and 0.4 as the cutoff values and defined three risk groups as low, medium, and high risk groups, corresponding to the scores of $<388$ points, $388-435$ points, and $>435$ points on the graphical nomogram. In this study, more than one-third of the patients were classified into the low risk 
TABLE 2 | Univariate analysis of possible risk factors for POH after AADS in the training set.

\begin{tabular}{|c|c|c|c|}
\hline Characteristics & $\begin{array}{c}\text { Without POH } \\
n=756(\%)\end{array}$ & $\begin{array}{l}\text { With POH } \\
n=277(\%)\end{array}$ & $P$ value \\
\hline \multicolumn{4}{|l|}{ Demographics } \\
\hline Age (years) & $51.92 \pm 11.33$ & $47.09 \pm 11.46$ & $<0.001$ \\
\hline Body mass index $\left(\mathrm{kg} / \mathrm{m}^{2}\right)$ & $25.34 \pm 3.58$ & $25.13 \pm 3.84$ & 0.409 \\
\hline \multicolumn{4}{|l|}{ Underlying conditions } \\
\hline Hypertension & $534(70.6)$ & $162(58.5)$ & $<0.001$ \\
\hline Diabetes mellitus & $29(3.8)$ & $13(4.7)$ & 0.537 \\
\hline Chronic headache history & $68(9.0)$ & $63(22.7)$ & $<0.001$ \\
\hline Chronic obstructive pulmonary disease & $12(1.6)$ & $1(0.4)$ & 0.117 \\
\hline Atrial fibrillation & $9(1.2)$ & $1(0.4)$ & 0.228 \\
\hline Cardiac surgery history & $42(5.6)$ & $28(10.1)$ & 0.010 \\
\hline General surgery history & $156(20.6)$ & $51(18.4)$ & 0.429 \\
\hline Pulmonary artery hypertension & $21(2.8)$ & $7(2.5)$ & 0.826 \\
\hline Pericardial effusion & $191(25.3)$ & $80(28.9)$ & 0.242 \\
\hline NYHA class III-IV & $65(8.6)$ & $22(7.9)$ & 0.737 \\
\hline Left ventricular ejection fraction (\%) & $62(59,65)$ & $61(59,65)$ & 0.336 \\
\hline \multicolumn{4}{|l|}{ Laboratory values } \\
\hline White blood cell count $\left(\times 10^{9} / L\right)$ & $9.97(7.42,12.37)$ & $10.45(7.92,14.24)$ & 0.002 \\
\hline Red blood cell count $\left(\times 10^{12} / L\right)$ & $4.29(3.86,4.63)$ & $4.22(3.77,4.62)$ & 0.506 \\
\hline Hemoglobin (g/l) & $130(116,140)$ & $128(114,141)$ & 0.272 \\
\hline Cardiopulmonary bypass time (minutes) & $193(159,234)$ & $243(198,288)$ & $<0.001$ \\
\hline Aortic cross clamp time (minutes) & $112(92,137)$ & $138(111,168)$ & $<0.001$ \\
\hline Deep hypothermic circulatory arrest & $392(51.9)$ & $219(79.1)$ & 0.807 \\
\hline Transfusion of red blood cells (units) & $4(2,5)$ & $6(3,7)$ & $<0.001$ \\
\hline
\end{tabular}

AADS, Stanford type A acute aortic dissection surgery; $\mathrm{POH}$, postoperative headache.

group, about two-fifths into the medium risk group, and about a quarter into the high risk group. We compared the predicted probabilities and the observed probabilities in the training and validation sets between the three risk groups (Figure 5). No significant difference was observed between the predicted and actual probabilities in the same risk interval $(P>0.05)$ and it differed significantly between different risk intervals $(P<0.05)$, which indicated good consistency and reasonable division.

\section{Outcomes}

The overall mortality rate of the included patients was $8.7 \%$, with a rate of $6.6 \%$ in patients without $\mathrm{POH}$ vs. $15.0 \%$ in those with $\mathrm{POH}$ [odds ratio $(\mathrm{OR})=2.510,95 \% \mathrm{CI}, 1.735-3.631$; $P<0.001)$. We also observed significantly higher probabilities of readmission to ICU, reintubation and tracheotomy, and significantly longer postoperative ICU and hospital stay in patients with $\mathrm{POH}$. Details of the comparison in patients with and without $\mathrm{POH}$ after AADS are presented in Table 6.

\section{DISCUSSION}

$\mathrm{POH}$ has been reported to be an indicator of increased risk of mortality and averse outcomes $(3,5,6)$, which was consistent with the present study. The overall incidence of POH after AADS 
TABLE 3 | Multivariate analysis of independent risk factors for POH after AADS.

\begin{tabular}{|c|c|c|c|c|}
\hline Characteristics & Coefficient & Standard error & OR $(95 \% \mathrm{Cl})$ & $P$ value \\
\hline Female sex & 1.082 & 0.247 & $2.951(1.817-4.792)$ & $<0.001$ \\
\hline Age (years) & -0.052 & 0.008 & $0.950(0.935-0.965)$ & $<0.001$ \\
\hline Smoking history & 1.163 & 0.210 & $3.201(2.121-4.831)$ & $<0.001$ \\
\hline Chronic headache history & 1.235 & 0.239 & $3.437(2.150-5.495)$ & $<0.001$ \\
\hline Cerebrovascular disease & 0.956 & 0.222 & $2.602(1.685-4.019)$ & $<0.001$ \\
\hline Cardiopulmonary bypass time (min) & 0.011 & 0.002 & $1.011(1.008-1.014)$ & $<0.001$ \\
\hline Intraoperative transfusion of RBC (units) & 0.248 & 0.042 & $1.281(1.181-1.390)$ & $<0.001$ \\
\hline Deep hypothermic circulatory arrest & 1.283 & 0.195 & 3.607 (2.459-5.290) & $<0.001$ \\
\hline Constant & -4.134 & 0.559 & 0.016 & $<0.001$ \\
\hline
\end{tabular}

AADS, Stanford type A acute aortic dissection surgery; Cl, confidence interval; OR, odds ratio; POH, postoperative headache; RBC, red blood cell.

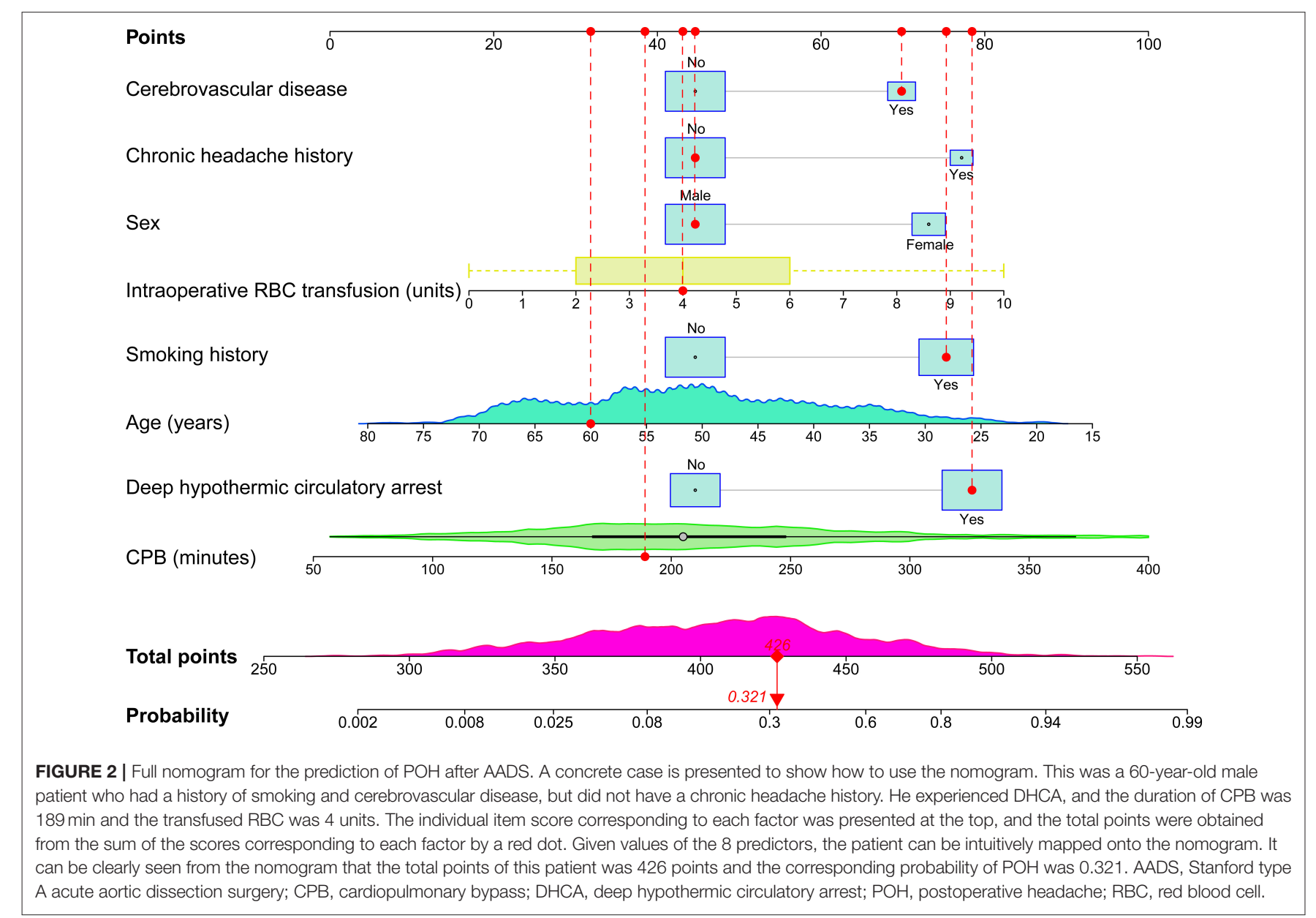

was $25.7 \%$ and the mortality was $8.7 \%$. However, compared with patients without $\mathrm{POH}$, the mortality was significantly higher in patients who suffered from POH. Moreover, we observed significantly higher probability of other adverse outcomes such as tracheotomy and reintubation in these patients. The higher risk of adverse outcomes and mortality stressed the need of identifying independent risk factors and high-risk patients of POH after AADS.
Globally, various studies focused on identifying predictors for $\mathrm{POH}$ have been carried out in patients undergoing other surgeries $(6,8-10,12)$, however, studies conducted in patients undergoing AADS are still lacking. To our knowledge, our work is the first report that describes the incidence, predictors, and outcomes of $\mathrm{POH}$ after AADS, and is the first attempt to construct and validate clinical prediction models in this area worldwide. In this study, 

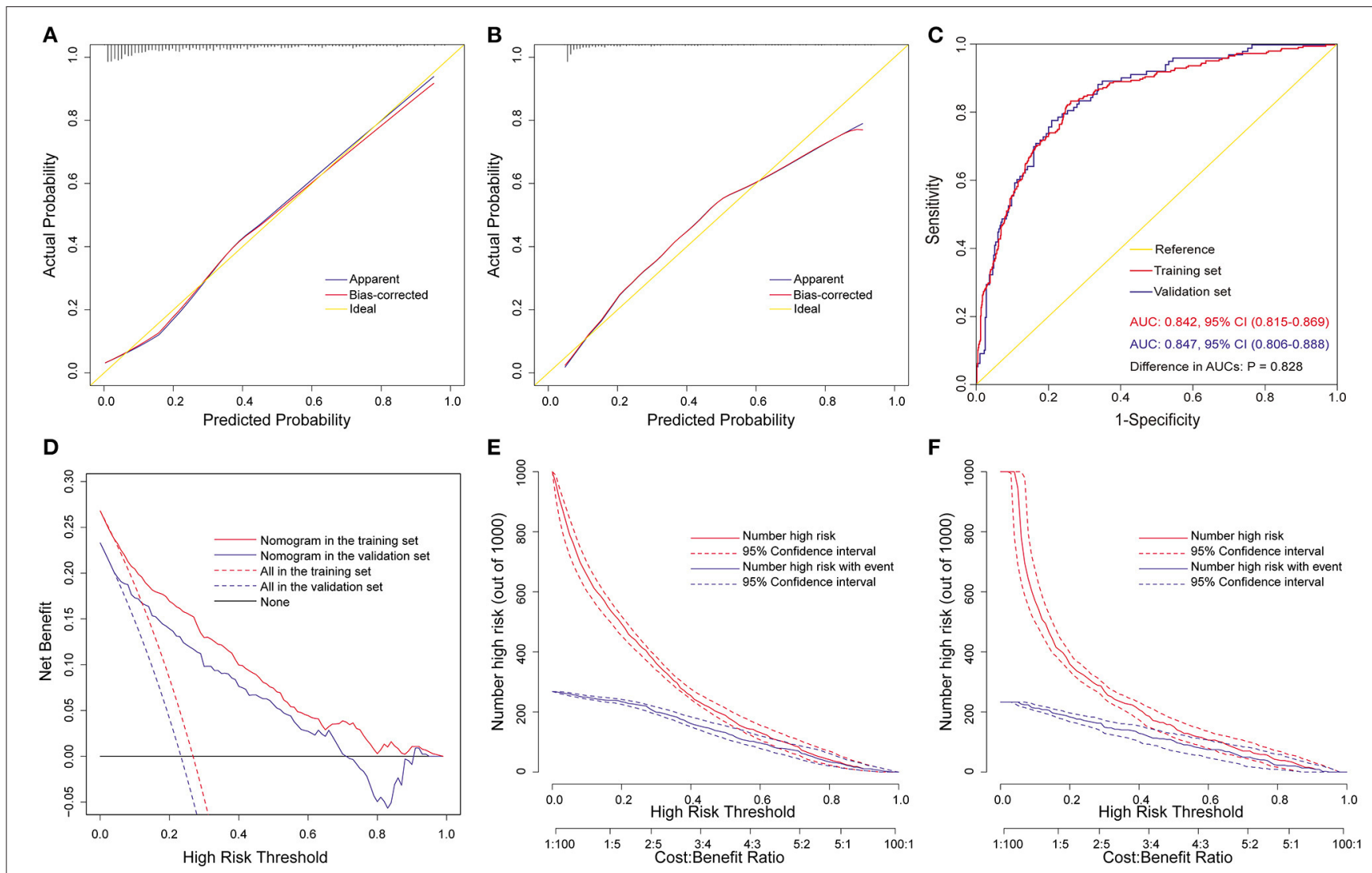

FIGURE 3 | Assessment of the full nomogram model for POH after AADS. Calibration plots in the training set (A) and the validation set (B), ROC curves in the two sets (C), decision curves in the two sets (D), and clinical impact curves in the training set (E) and the validation set (F). AADS, Stanford type A acute aortic dissection surgery; AUC, area under the receiver operating characteristic curve; $\mathrm{Cl}$, confidence interval; $\mathrm{POH}$, postoperative headache; ROC, receiver operating characteristic curve.

TABLE 4 | Multivariate analysis of preoperative independent risk factors for $\mathrm{POH}$ after AADS.

\begin{tabular}{|c|c|c|c|c|}
\hline Characteristics & Coefficient & $\begin{array}{l}\text { Standard } \\
\text { error }\end{array}$ & OR (95\% Cl) & $P$ value \\
\hline Platelet count $\left(\times 10^{9} / \mathrm{L}\right)$ & -0.004 & 0.001 & 0.996 (0.994-0.998) & 0.002 \\
\hline Female sex & 1.332 & 0.228 & 3.789 (2.423-5.927) & $<0.001$ \\
\hline Age (years) & -0.045 & 0.007 & $0.956(0.942-0.969)$ & $<0.001$ \\
\hline Smoking history & 1.291 & 0.194 & $3.636(2.488-5.313)$ & $<0.001$ \\
\hline Cerebrovascular disease & 0.949 & 0.198 & $2.584(1.753-3.808)$ & $<0.001$ \\
\hline Chronic headache history & 1.060 & 0.217 & $2.888(1.888-4.418)$ & $<0.001$ \\
\hline $\begin{array}{l}\text { White blood cell count } \\
\left(\times 10^{9} / L\right)\end{array}$ & 0.084 & 0.020 & $1.087(1.045-1.132)$ & $<0.001$ \\
\hline Constant & -1.610 & 0.625 & 0.200 & 0.010 \\
\hline
\end{tabular}

AADS, Stanford type A acute aortic dissection surgery; Cl, confidence interval; OR, odds ratio; $\mathrm{POH}$, postoperative headache.

we developed and validated a full nomogram model and a preoperative nomogram model using clinical data of 1,476 patients who underwent AADS in four tertiary care centers. The former was constructed based on five preoperative and three intraoperative predictors and the latter was based on seven preoperative predictors. Both nomogram models indicated good calibration, discrimination, and clinical usefulness. Three risk groups were finally divided to facilitate clinical application based on the full nomogram model and clinical practice.

Younger age and female sex have been reported to be associated with the development of $\mathrm{POH}$ in various surgeries $(8,9,11,14-16)$, which was consistent with our results. Droby and colleagues conducted a prospective study in patients who experienced post-lumbar puncture and found that compared to participants who did not develop a $\mathrm{POH}$, patients that developed a POH were younger $(P=0.033)(17)$. Bitargil and colleagues conducted a respective study in patients undergoing endothermal ablation of the greater saphenous vein under spinal anesthesia and reported that female patients suffered from significantly more headaches than males ( 27 vs. $10 \%, P=0.013$ ) (9). Takenaka and colleagues conducted a large-scale study in patients who underwent primary lumbar spine surgery and reported that $\mathrm{POH}$ were more frequently observed in females than in males $(\mathrm{OR}=2.70, P=0.001)$ and advanced age was a significant protective factor for $\mathrm{POH}(\mathrm{OR}=0.70, P<$ 0.001) (14). 


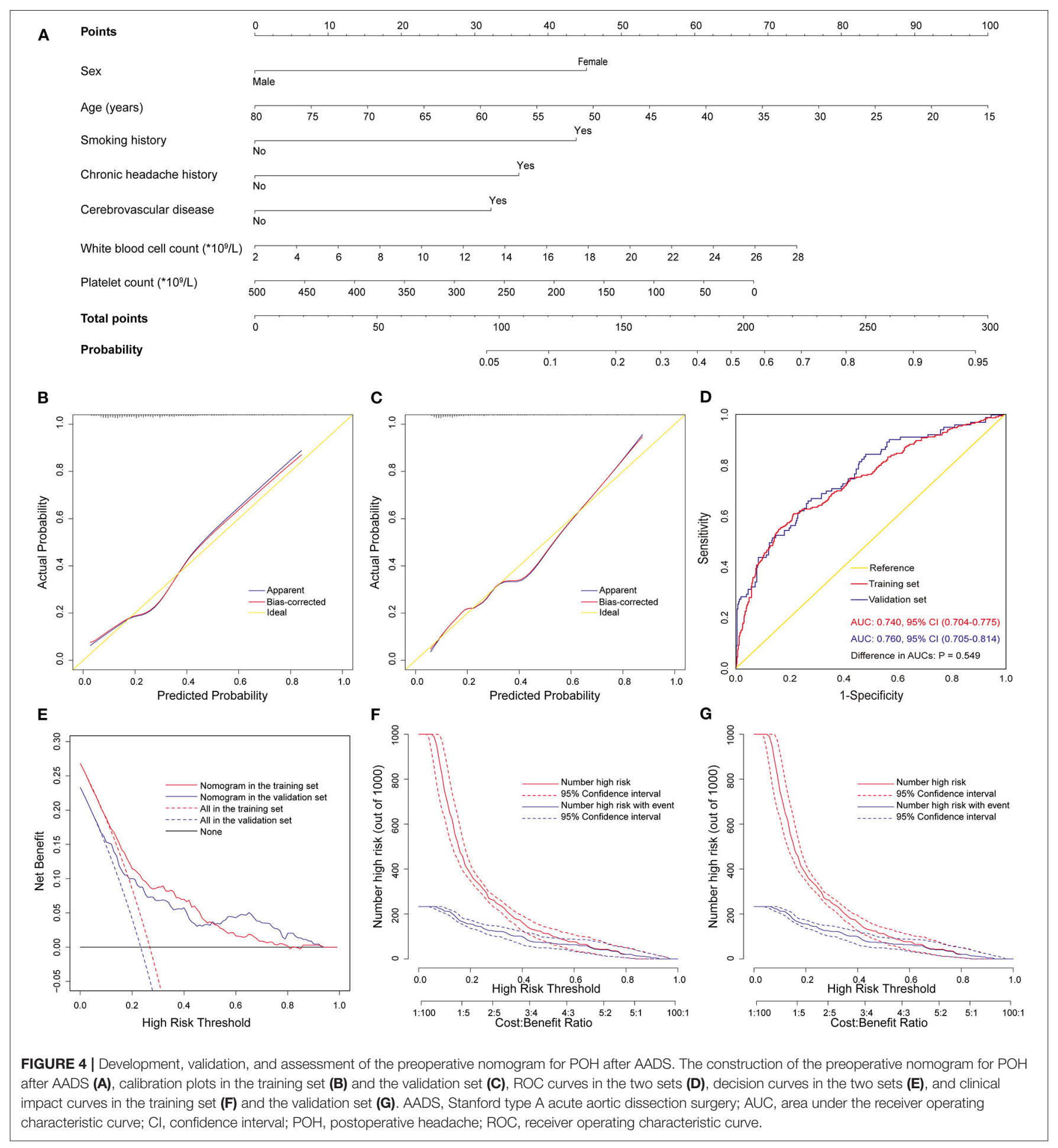

The sex- and age-related difference in $\mathrm{POH}$ are likely multifactorial. Recently, several potential mechanisms have been posited such as hormones influence, pain perception, and psychosocial factors (18). Fluctuating hormone levels during the menstrual cycle and hormone-related differences in cerebrovascular reactivity have long been blamed for the development of kinds of headaches (19-21). In terms of pain perception, it is believed that females and younger patients are more sensitive than males and older patients (22). In addition, compared to males and older patients, females and younger patients prefer to interpret, recall and report physical discomforts as symptoms such as pain, which may be due to the fact that 
TABLE 5 | Risk intervals of POH based on the nomogram.

\begin{tabular}{lccc}
\hline Risk intervals & $\begin{array}{c}\text { Low risk } \\
\text { (<388 points) }\end{array}$ & $\begin{array}{c}\text { Medium risk } \\
\mathbf{( 3 8 8 - 4 3 5} \text { points) }\end{array}$ & $\begin{array}{c}\text { High risk } \\
\mathbf{( > 4 3 5} \text { points) }\end{array}$ \\
\hline $\begin{array}{l}\text { Estimated probability (\%) } \\
\text { Observed probability, }\end{array}$ & $4.7(2.8-6.5)$ & $21.2(17.9-24.5)$ & $62.7(57.7-67.6)$ \\
$\%(95 \%$ Cl) & & $10-40$ & $>40$ \\
No. of patients (\%) & $515(34.9)$ & $594(40.2)$ & $367(24.9)$
\end{tabular}

$\mathrm{Cl}$, confidence interval; $\mathrm{POH}$, postoperative headache.

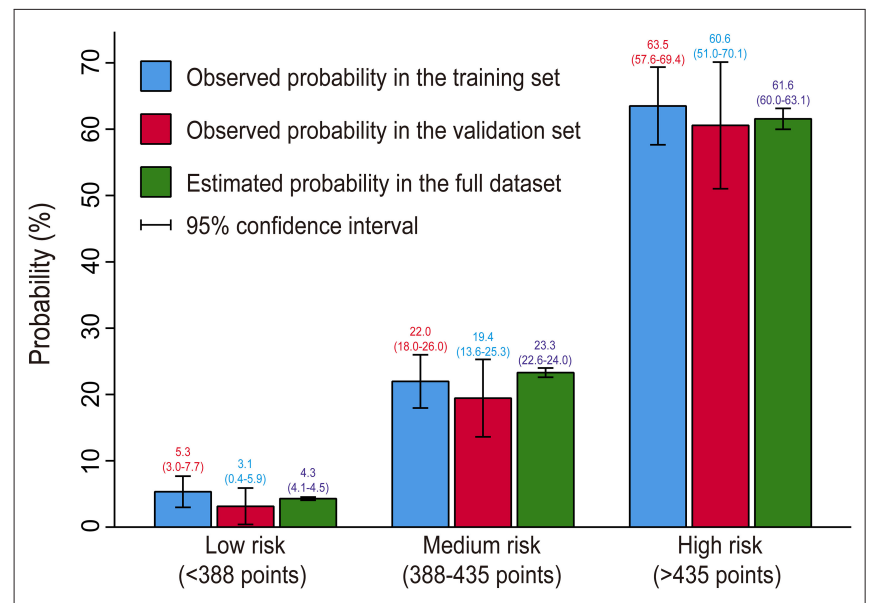

FIGURE 5 | Bar chart showing the consistency between predicted and observed probabilities. No significant difference was observed between the predicted and actual probabilities in the same risk interval $(P>0.05)$ and it differed significantly between different risk intervals $(P<0.05)$, indicating good consistency and reasonable division.

TABLE 6 | Postoperative variables in patients with and without POH after AADS.

\begin{tabular}{lcccc}
\hline Variables & All patients & Without POH & With POH & $P$ value \\
& $\boldsymbol{n}=\mathbf{1 , 4 7 6}(\%)$ & $\boldsymbol{n = 1 , 0 9 6 ( \% )}$ & $\boldsymbol{n}=\mathbf{3 8 0}(\%)$ & \\
\hline Reintubation & $216(14.6)$ & $122(11.1)$ & $94(24.7)$ & $<0.001$ \\
Tracheotomy & $165(11.2)$ & $88(8.0)$ & $77(20.3)$ & $<0.001$ \\
Readmission to ICU & $132(8.9)$ & $84(7.7)$ & $48(12.6)$ & 0.003 \\
ICU stay (days) & $7(5,11)$ & $6(5,9)$ & $9(6,15)$ & $<0.001$ \\
Hospital stay (days) & $21(17,27)$ & $20(16,26)$ & $24(19,31)$ & $<0.001$ \\
Mortality & $129(8.7)$ & $72(6.6)$ & $57(15.0)$ & $<0.001$
\end{tabular}

AADS, Stanford type A acute aortic dissection surgery; ICU, intensive care unit; $P O H$, postoperative headache.

it is more socially acceptable to express such emotions in those patients (16).

Chronic headache history was identified as an independent predictor for $\mathrm{POH}$ after AADS by multivariate analysis, which was in agreement with previous results in the literature (12, $15,23,24)$. Valentinis and colleagues conducted a prospective cohort study in patients who were operated on for intracranial tumors and reported that a longstanding headache history was the only significant independent intrapersonal predictor for $\mathrm{POH}$ $(\mathrm{OR}=3.07, P=0.01)(23)$. Ryzenman and colleagues conducted a large prospective cohort study in patients undergoing acoustic neuroma surgery to investigate the incidence and risk factors of $\mathrm{POH}$ and its effects on physical and psychosocial function (15). They found that preoperative headache was independently associated with the development of $\mathrm{POH}(\mathrm{OR}=1.4, P<0.01)$ and patients who suffered from preoperative headache had more multiple occurrences of POH daily. Furthermore, Yabuki and colleagues reported that patients with preoperative headache had higher pain levels, higher neuropathic pain symptoms, and poorer quality of life (25). Hence, we believe that it may be an appropriate option to take prophylactic drugs to reduce the risk of POH for patients who had a chronic headache history (26).

Several other preoperative independent predictors for $\mathrm{POH}$ were also identified in our analysis, including cerebrovascular disease, smoking, WBC and platelet count. Headaches are common accompanying symptoms of cerebrovascular diseases and headaches attributed to ischemic strokes and transient ischemic attacks occur frequently. Oliveira and colleagues reported that the prevalence of headaches attributed to transient ischemic attacks and ischemic strokes were respectively $7.4-34 \%$ and $26-36 \%$ (27). A prospective study conducted by Matsota and colleagues indicated that smoking history was independently associated with the development of $\mathrm{POH}$ in patients undergoing elective surgery patients $(\mathrm{OR}=1.74, P=0.006)$ (8). Although the exact linkage between smoking and headache remains to unknown at present, it is undeniable that tobacco exposure is in some manner related to cluster headache (28). Given numerous negative health effects, decreased tobacco exposure and smoking cessation should be recommended in hopes of reducing disability and improving functionality (29).

The elevation of WBC count as a predictor for $\mathrm{POH}$ has been identified in previous study, which may relate to the acute phase systemic inflammatory response (24). Yazar and colleagues carried out a prospective study to investigate the role of inflammation and oxidative stress in the etiology of migraine (30). They found that the neutrophil, neutrophil/lymphocyte, monocyte/lymphocyte and platelet/lymphocyte ratios were higher in patients with migraine than patients without that $(P<0.05)$. The serum C-reactive protein, neutrophil, neutrophil/lymphocyte, monocyte/lymphocyte, and C-reactive protein /albumin ratios were higher during migraine attack periods $(P<0.05)$. Guo and colleagues conducted a retrospective study to explore prognostic factors for permanent neurological dysfunction after total aortic arch replacement with regional cerebral oxygen saturation monitoring. By multiple logistic regression analysis, they found that preoperative low platelet count was an independent predictor for postoperative neurological complications, which may be associated with platelet consumption coagulopathy (31). Siewert and colleagues conducted a genetic correlation analysis to explore risk factors for migraine headache using cross-trait linkage disequilibrium score regression and tested for potential causality between migraine and those phenotypes using Mendelian randomization (32). They found that migraine headache had genetic correlations with various traits including cardiovascular disease, smoking status, WBC count and platelet count. 
Besides the preoperative predictors mentioned above, three intraoperative predictors for $\mathrm{POH}$ after AADS were also identified in this study, including RBC transfusion, $\mathrm{CPB}$ time and the use of DHCA. Although RBC transfusion can be lifesaving during cardiovascular surgery, massive transfusion has been confirmed to relate to various adverse events $(33,34)$. Arngrim and colleagues conducted a MRI spectroscopy and angiography study finding that experimental hypoxia was associated with headache attacks (35). Accordingly, we hypothesize that the reduced capacity of oxygen-carrying of the transfused RBCs which may lead to insufficient oxygen supply to the brain may be one of the responsible causes of $\mathrm{POH}$. Furthermore, massive transfusion of RBC is often due to massive blood loss, which may also result in insufficient oxygen supply due to blood dilution and the reduction of active RBC and hemoglobin. Recently, a restrictive blood transfusion strategy has been especially recommended in clinical practice guidelines to prevent the development of various adverse events $(36,37)$.

It can be easily understood that CPB time is independently associated with the development of $\mathrm{POH}$ after AADS. On the one hand, longer duration of $\mathrm{CPB}$ often indicates longer duration of the whole surgery, longer forced position, and more intake of anesthetic agents, which have been reported to be significantly related to the development of $\mathrm{POH}(8,38)$. On the other hand, the $\mathrm{CPB}$ process itself can result in brain injury and $\mathrm{POH}$ in many ways, including cerebral edema, embolism, hemodilution, and hypoxia (39-41). A systematic review conducted by Caldas and colleagues indicated that the development of neurological complications in patients who underwent cardiac surgery may be partially caused by the damage of cerebral autoregulation during CPB (40). Therefore, it may be effective to decrease the incidence of $\mathrm{POH}$ and other neurological complications through better brain protection strategy during CPB (41).

The use of DHCA has long been considered to be the standard neuroprotection strategy in patients undergoing AADS, however, this technique remains related to significant risk of brain damage and complications (42), which was again confirmed by this study. To reduce the risk of brain damage, continuous cerebral perfusion techniques have been proposed these years, including antegrade cerebral perfusion via the right subclavian artery only or with selective perfusion of both the carotid arteries and retrograde cerebral perfusion via the venous system. Using the UK National Adult Cardiac Surgical Audit, Benedetto and colleagues investigated the association between neuroprotective strategies and clinical outcomes in patients undergoing AADS (43). They found that compared to DHCA, the use of unilateral and bilateral antegrade cerebral perfusion was related to a decreased risk of death and cerebrovascular accident. Nonetheless, no consensus has been reached on which neuroprotective strategy should be preferred and there exists significant variation in clinical practice (44-47).

The nomograms may be helpful for risk evaluation, early prevention, surgeon-patient communication, and clinical decision-making. Taking appropriate strategies based on the nomograms may obtain more clinical net benefits. Besides preoperative precautions, preventive efforts during operations also make sense. Bezov and colleagues concluded that operator experience was a modifiable risk factors for POH (18). Benedetto and colleagues reported that high-volume surgeons, cardiac centers, and intraoperative factors were strong determinants of clinical outcomes after AADS (1).

There are several limitations in this study. First, the study was retrospectively designed and $\mathrm{POH}$ was diagnosed on the basis of medical records. Thus, we cannot assure that all the patients with $\mathrm{POH}$ were recorded in the database, which may result in an underestimation of the true morbidity. Second, some factors that may significantly relate to $\mathrm{POH}$ were not available in our analysis, such as operator experience. Nevertheless, the nomograms had reasonable performance in predictive capability, calibration, and clinical usefulness. Third, $\mathrm{POH}$ was the primary endpoint, but the evaluation of the types and severity of $\mathrm{POH}$ was not available because of the retrospective limitations. Prospective studies with more explicit classification of the types and severity of $\mathrm{POH}$ may make more sense in future work.

\section{CONCLUSIONS}

To our knowledge, this is the first study describing the incidence, risk factors and outcomes of $\mathrm{POH}$ in patients undergoing AADS. $\mathrm{POH}$ was prevalent in our results, and the mortality and other adverse outcomes increased significantly in patients with $\mathrm{POH}$. We constructed a full nomogram using five preoperative and three intraoperative predictors and a preoperative nomogram using seven preoperative predictors. Both nomograms demonstrated good calibration, discrimination, and clinical utility. We further defined three risk groups to facilitate clinical application. These findings may be helpful for risk evaluation, surgeon-patient communication, clinical decision-making, and early prevention.

\section{DATA AVAILABILITY STATEMENT}

The datasets presented in this article are not readily available because of patient confidentiality. Requests to access the datasets should be directed to the corresponding author.

\section{ETHICS STATEMENT}

The studies involving human participants were reviewed and approved by the Ethics Committee of Tongji Medical College of Huazhong University of Science and Technology (IORG No. IORG0003571). Written informed consent for participation was not required for this study in accordance with the national legislation and the institutional requirements.

\section{AUTHOR CONTRIBUTIONS}

$\mathrm{XD}, \mathrm{XH}, \mathrm{PY}$, and AZ: conception and design. XD, PY, HW, and SL: administrative support. $\mathrm{XD}, \mathrm{XH}, \mathrm{HW}$, and $\mathrm{AZ}$ : provision of study materials or patients. DW, JW, FX, $\mathrm{XL}, \mathrm{SL}, \mathrm{RL}, \mathrm{YS}, \mathrm{PY}$, and JL: collection and assembly of 
data. DW, SL, and $\mathrm{XH}$ : data analysis and interpretation. All authors: manuscript writing and final approval of manuscript.

\section{FUNDING}

This work was supported by the National Natural Science Foundation of China (Grant Nos. 81800413, 81974048, and 81801586).

\section{ACKNOWLEDGMENTS}

The authors would like to thank Dr. John S for the language help.

\section{SUPPLEMENTARY MATERIAL}

The Supplementary Material for this article can be found online at: https://www.frontiersin.org/articles/10.3389/fcvm. 2021.781137/full\#supplementary-material

Supplementary Figure 1 | Screenshots of the interactive web-based dynamic nomogram created for the full predictive model of $\mathrm{POH}$ after AADS. Squares represent the point estimates for a given set of conditions, and the bars surrounding them reflect the $95 \% \mathrm{Cls}$. In the screenshots, we present the probabilities and corresponding $95 \%$ Cls of $\mathrm{POH}$ of 10 different patients (A); when click the square, the information of the patient and corresponding risk are presented $\mathbf{( B )}$; all the information of the patients can be acquired by clicking the "Numerical Summary" (C); and the information of the model can be acquired by clicking the "Model Summary" (D).

\section{REFERENCES}

1. Benedetto U, Dimagli A, Kaura A, Sinha S, Mariscalco G, Krasopoulos G, et al. Determinants of outcomes following surgery for type A acute aortic dissection: the UK National Adult Cardiac Surgical Audit. Eur Heart J. (2021). doi: 10.1093/eurheartj/ehab586. [Epub ahead of print].

2. Malaisrie SC, Szeto WY, Halas M, Girardi LN, Coselli JS, Sundt TR, et al. 2021 The American association for thoracic surgery expert consensus document: surgical treatment of acute type A aortic dissection. J Thorac Cardiovasc Surg. (2021) 162:735-58. doi: 10.1016/j.jtcvs.2021.04.053

3. Sabab A, Sandhu J, Bacchi S, Jukes A, Zacest A. Postoperative headache following treatment of vestibular schwannoma: a literature review. J Clin Neurosci. (2018) 52:26-31. doi: 10.1016/j.jocn.2018.04.003

4. Jang MK, Oh EG, Lee H, Kim EH, Kim S. Postoperative symptoms and quality of life in pituitary macroadenomas patients. J Neurosci Nurs. (2020) 52:30-6. doi: 10.1097/JNN.0000000000000483

5. Rocha-Filho PAS. Post-craniotomy headache: a clinical view with a focus on the persistent form. Headache. (2015) 55:733-8. doi: 10.1111/head.12563

6. Jang MK, Park CG, Jang S, Kim EH. Prevalence and impact of postoperative headaches in nonfunctioning pituitary macroadenoma patients: a longitudinal cohort study. World Neurosurg. (2020) 133:e633-9. doi: 10.1016/j.wneu.2019.09.123

7. Pogoda L, Nijdam JS, Smeeing DPJ, Voormolen EHJ, Ziylan F, Thomeer HGXM. Postoperative headache after surgical treatment of cerebellopontine angle tumors: a systematic review. Eur Arch Oto-Rhino-L. (2021). doi: 10.1007/s00405-021-06627-6

8. Matsota PK, Christodoulopoulou TC, Batistaki CZ, Arvaniti CC, Voumvourakis KI, Kostopanagiotou GG. Factors associated with the presence of postoperative headache in elective surgery patients: a prospective single center cohort study. J Anesth. (2017) 31:225-36. doi: 10.1007/s00540-016-2285-z

9. Bitargil M, El Kiliç H. Our experience regarding patients with headache, vomiting, and urinary retention following endothermal ablation of the greater saphenous vein under spinal anesthesia: gender type, age interval, and procedural risk factors are important. Vascular. (2020) 28:5916. doi: 10.1177/1708538120911302

10. Yilmaz G, Akca A, Kiyak H, Salihoglu Z. Elevation in optic nerve sheath diameter due to the pneumoperitoneum and trendelenburg is associated to postoperative nausea, vomiting and headache in patients undergoing laparoscopic hysterectomy. Minerva Anestesiol. (2020) 86:2706. doi: 10.23736/S0375-9393.19.13920-X

11. Liang B, Shetty SR, Omay SB, Almeida JP, Ni S, Chen Y, et al. Predictors and incidence of orthostatic headache associated with lumbar drain placement following endoscopic endonasal skull base surgery. Acta Neurochir. (2017) 159:1379-85. doi: 10.1007/s00701-017-3247-4

12. Gill PS, Guest C, Rabey PG, Buggy DJ. Perioperative headache and day case surgery. Eur J Anaesthesiol. (2003) 20:4013. doi: 10.1097/00003643-200305000-00009

13. DeLong ER, DeLong DM, Clarke-Pearson DL. Comparing the areas under two or more correlated receiver operating characteristic curves: a nonparametric approach. Biometrics. (1988) 44:837-45. doi: 10.2307/2531595

14. Takenaka S, Makino T, Sakai Y, Kashii M, Iwasaki M, Yoshikawa $\mathrm{H}$, et al. Prognostic impact of intra- and postoperative management of dural tear on postoperative complications in primary degenerative lumbar diseases. Bone Joint J. (2019) 101-B:1115-21. doi: 10.1302/0301-620X.101B9.BJJ-2019-0381.R1

15. Ryzenman JM, Pensak ML, Tew JJ. Headache: a quality of life analysis in a cohort of 1,657 patients undergoing acoustic neuroma surgery, results from the acoustic neuroma association. Laryngoscope. (2005) 115:70311. doi: 10.1097/01.mlg.0000161331.83224.c5

16. Myles PS, Hunt JO, Moloney JT. Postoperative minor complications: comparison between men and women. Anaesthesia. (1997) 52:3006. doi: 10.1111/j.1365-2044.1997.89-az0091.x

17. Droby A, Omer N, Gurevich T, Kestenbaum M, Mina Y, Cedarbaum JM, et al. Low cerebrospinal fluid volume and the risk for post-lumbar puncture headaches. J Neurol Sci. (2020) 417:117059. doi: 10.1016/j.jns.2020.117059

18. Bezov D, Lipton RB, Ashina S. Post-dural puncture headache: part I diagnosis, epidemiology, etiology, and pathophysiology. Headache. (2010) 50:114452. doi: 10.1111/j.1526-4610.2010.01699.x

19. Hipolito Rodrigues MA, Maitrot-Mantelet L, Plu-Bureau G, Gompel A. Migraine, hormones and the menopausal transition. Climacteric. (2018) 21:256-66. doi: 10.1080/13697137.2018.1439914

20. Gupta S, McCarson KE, Welch KMA, Berman NEJ. Mechanisms of pain modulation by sex hormones in migraine. Headache. (2011) 51:90522. doi: 10.1111/j.1526-4610.2011.01908.x

21. Anne MacGregor E. Oestrogen and attacks of migraine with and without aura. Lancet Neurol. (2004) 3:354-61. doi: 10.1016/S1474-4422(04)00768-9

22. Novais A, Monteiro S, Roque S, Correia-Neves M, Sousa N. How age, sex and genotype shape the stress response. Neurobiol Stress. (2017) 6:4456. doi: 10.1016/j.ynstr.2016.11.004

23. Valentinis L, Tuniz F, Valent F, Mucchiut M, Little D, Skrap M, et al. Headache attributed to intracranial tumours: a prospective cohort study. Cephalalgia. (2010) 30:389-98. doi: 10.1111/j.1468-2982.2009.01970.x

24. Wang D, Huang X, Wang H, Le S, Du X. Predictors and nomogram models for postoperative headache in patients undergoing heart valve surgery. $J$ Thorac Dis. (2021) 13:4236-49. doi: 10.21037/jtd-21-644

25. Yabuki S, Takatsuki K, Otani K, Nikaido T, Watanabe K, Kato K, et al. Headache in Patients with Cervical Spondylotic Myelopathy. Pain Res Manag. (2020) 2020:1-6. doi: 10.1155/2020/8856088

26. Evers S. Treatment of migraine with prophylactic drugs. Expert Opin Pharmacother. (2008) 9:2565-73. doi: 10.1517/14656566.9.15.2565

27. Oliveira F, Sampaio RP. Headaches attributed to ischemic stroke and transient ischemic attack. Headache. (2019) 59:469-76. doi: 10.1111/head.13478

28. Rozen TD. Linking cigarette smoking/tobacco exposure and cluster headache: a pathogenesis theory. Headache. (2018) 58:1096-112. doi: 10.1111/head.13338 
29. Taylor FR. Tobacco, nicotine, and headache. Headache. (2015) 55:102844. doi: 10.1111/head.12620

30. Yazar HO, Yazar T, Aygun A, Kaygisiz S, Kirbas D. Evaluation of simple inflammatory blood parameters in patients with migraine. Ir J Med Sci. (2020) 189:677-83. doi: 10.1007/s11845-019-02136-y

31. Yu Y, Lyu Y, Jin L, Xu L, Wang H, Hu Y, et al. Prognostic factors for permanent neurological dysfunction after total aortic arch replacement with regional cerebral oxygen saturation monitoring. Brain Behav. (2019) 9:e1309. doi: 10.1002/brb3.1309

32. Siewert KM, Klarin D, Damrauer SM, Chang K, Tsao PS, Assimes TL, et al. Cross-trait analyses with migraine reveal widespread pleiotropy and suggest a vascular component to migraine headache. Int J Epidemiol. (2020) 49:102231. doi: 10.1093/ije/dyaa154

33. Sultan I, Bianco V, Aranda-Michel E, Kilic A, Serna-Gallegos D, Navid F, et al. The use of blood and blood products in aortic surgery is associated with adverse outcomes. J Thorac Cardiovasc Surg. (2021). doi: 10.1016/j.jtcvs.2021.02.096. [Epub ahead of print].

34. Wang D, Huang X, Wang H, Le S, Yang H, Wang F, et al. Risk factors for postoperative pneumonia after cardiac surgery: a prediction model. J Thorac Dis. (2021) 13:2351-62. doi: 10.21037/jtd-20-3586

35. Arngrim N, Schytz HW, Britze J, Amin FM, Vestergaard MB, Hougaard A, et al. Migraine induced by hypoxia: an MRI spectroscopy and angiography study. Brain. (2016) 139:723-37. doi: 10.1093/brain/awv359

36. Carson JL, Guyatt G, Heddle NM, Grossman BJ, Cohn CS, Fung $\mathrm{MK}$, et al. Clinical practice guidelines from the AABB: red blood cell transfusion thresholds and storage. JAMA. (2016) 316:2025-35. doi: 10.1001/jama.2016.9185

37. Jeffrey L, Apfelbaum MD, Gregory A, Nuttall MD, Richard T Connis PD, D CRHM, et al. Practice guidelines for perioperative blood management: an updated report by the American society of anesthesiologists task force on perioperative blood management*. Anesthesiology. (2015) 122:24175. doi: 10.1097/ALN.0000000000000463

38. de Oliveira Ribeiro MDC, Pereira CU, Sallum AM, Martins-Filho PRS, DeSantana JM, Da Silva Nunes $\mathrm{M}$, et al. Immediate post-craniotomy headache. Cephalalgia. (2013) 33:897-905. doi: 10.1177/0333102413479833

39. Zhu S, Sai X, Lin J, Deng G, Zhao M, Nasser MI, et al. Mechanisms of perioperative brain damage in children with congenital heart disease. Biomed Pharmacother. (2020) 132:110957. doi: 10.1016/j.biopha.2020.110957

40. Caldas JR, Haunton VJ, Panerai RB, Hajjar LA, Robinson TG. Cerebral autoregulation in cardiopulmonary bypass surgery: a systematic review. Interact Cardiov Th. (2018) 26:494-503. doi: 10.1093/icvts/ivx357

41. Salameh A, Dhein S, Dahnert I, Klein N. Neuroprotective strategies during cardiac surgery with cardiopulmonary bypass. Int J Mol Sci. (2016) 17:1945. doi: 10.3390/ijms17111945
42. Algarni KD, Yanagawa B, Rao V, Yau TM. Profound hypothermia compared with moderate hypothermia in repair of acute type A aortic dissection. J Thorac Cardiovasc Surg. (2014) 148:2888-94. doi: 10.1016/j.jtcvs.2014. 01.020

43. Benedetto U, Dimagli A, Cooper G, Uppal R, Mariscalco G, Krasopoulos G, et al. Neuroprotective strategies in acute aortic dissection: an analysis of the UK national adult cardiac surgical audit. Eur J Cardiothorac Surg. (2021). 60:1437-44. doi: 10.1093/ejcts/ ezab192

44. Piperata A, Watanabe M, Pernot M, Metras A, Kalscheuer G, Avesani M, et al. Unilateral versus bilateral cerebral perfusion during aortic surgery for acute type A aortic dissection: a multicentre study. Eur J Cardiothorac Surg. (2021). doi: 10.1093/ejcts/ezab341. [Epub ahead of print].

45. Norton EL, Wu X, Kim KM, Patel HJ, Deeb GM, Yang B. Unilateral is comparable to bilateral antegrade cerebral perfusion in acute type A aortic dissection repair. J Thorac Cardiovasc Surg. (2020) 160:61725. doi: 10.1016/j.jtcvs.2019.07.108

46. Liu Z, Wang C, Zhang X, Wu S, Fang C, Pang X. Effect of different types of cerebral perfusion for acute type A aortic dissection undergoing aortic arch procedure, unilateral versus bilateral. BMC Surg. (2020) 20:286. doi: 10.1186/s12893-020-00957-8

47. Angeloni E, Melina G, Refice SK, Roscitano A, Capuano F, Comito C, et al. Unilateral versus bilateral antegrade cerebral protection during aortic surgery: an updated meta-analysis. Ann Thorac Surg. (2015) 99:202431. doi: 10.1016/j.athoracsur.2015.01.070

Conflict of Interest: The authors declare that the research was conducted in the absence of any commercial or financial relationships that could be construed as a potential conflict of interest.

Publisher's Note: All claims expressed in this article are solely those of the authors and do not necessarily represent those of their affiliated organizations, or those of the publisher, the editors and the reviewers. Any product that may be evaluated in this article, or claim that may be made by its manufacturer, is not guaranteed or endorsed by the publisher.

Copyright (C) 2021 Wang, Le, Luo, Chen, Li, Wu, Song, Xie, Li, Wang, Huang, Ye, Du and Zhang. This is an open-access article distributed under the terms of the Creative Commons Attribution License (CC BY). The use, distribution or reproduction in other forums is permitted, provided the original author(s) and the copyright owner(s) are credited and that the original publication in this journal is cited, in accordance with accepted academic practice. No use, distribution or reproduction is permitted which does not comply with these terms. 